\title{
PEQUEÑAS CENTRALES HIDROELÉCTRICAS \\ Una mirada a la experiencia africana en los años ochenta
}

\author{
Jaime Loboguerrero Uscátegui \\ Profesor titular, Deptartamento de Ingeniería Mecánica, Universidad de los Andes. \\ contacto:jlobogue@uniandes.edu.co
}

Uno de los asuntos tratados a lo largo de esta edición fue la provisión de energía en las zonas rurales colombianas mediante la utilización de paneles solares, turbinas eólicas o pequeñas centrales hidroeléctricas, que, además de ser bastante eficientes y asequibles, garantizan la preservación del medio ambiente pues sus emisiones contaminantes son mínimas.

Por tal razón, para esta edición de la Revista de Ingeniería hemos decidido replicar en la Sección Memoria un artículo del profesor Jaime Loboguerrero, publicado por primera vez en marzo de 1982. En este, el profesor Loboguerrero hace un balance de la utilidad que tienen las pequeñas centrales hidroeléctricas en el contexto africano.

\section{Introducción}

El término "pequeña central hidroeléctrica" se refiere a las centrales hidroeléctricas con capacidad instalada en el rango de $0.2 \mathrm{~kW}$ (las cuales suministran a hogares rurales individuales) hasta $500 \mathrm{~kW}$ (suministran a aldeas o actividades industriales, o ambas). Las centrales dentro de este rango, aunque son similares en principio a las mini-centrales hidroeléctricas (10 MW) y a las grandes centrales hidroeléctricas (100 MW), requieren un enfoque diferente en su diseño, desarrollo y operación. Su pequeña dimensión y comparativa menor inversión de capital por central imposibilita estudios de ingeniería costosos y pericia técnica especializada para el manejo y el mantenimiento.

Los ingenieros y planificadores en el ámbito de la energía hidroeléctrica están generalmente entrenados para pensar en términos de centrales enormes. Sin embargo, en las pequeñas hidroeléctricas el objetivo son muchas centrales diminutas ampliamente distribuidas, todas diferentes y sujetas a las condiciones cambiantes del sitio específico. Los problemas, por lo tanto, son más complicados.

En los proyectos grandes de energía se tiende a ignorar a los usuarios finales, quienes aparecen en los informes como estadísticas carentes de nombres. Aquellos implicados en las centrales medianas y pequeñas, en cambio, probablemente han de conocer y hablar con los usuarios finales quienes, muy seguramente, estarán involucrados en el proyecto desde el inicio. De hecho, para las centrales más pequeñas, el paquete de energía puede venderse como una pieza de equipo agrícola para que el usuario final lo instale por sí mismo. 




Pequeña central hidroeléctrica en Kenia, África. Fuente: Energytransition.de

\section{Tipos de proyectos de microhidroeléctrica}

Debido a su variedad, los proyectos de microhidroeléctrica son extremadamente difíciles de clasificar. Es posible utilizar clasificaciones técnicas, tales como la alta, media o baja presión. Asimismo, es posible definir los proyectos como aquellos que son aptos para recursos hídricos escasos y capital escaso, y aquellos que son aptos para recursos hídricos abundantes y capital escaso. Las microhidroeléctricas para países en desarrollo se encuentran en el punto medio y la tecnología ofrece una miríada de alternativas para cada caso.

En los sitios donde la demanda doméstica de energía es baja y los recursos hídricos son escasos, la única alternativa es el almacenamiento de energía; en los sitios donde los recursos hídricos son abundantes, un proyecto de agua fluyente podría ser el apropiado, pero también se debe tener en cuenta el almacenamiento de energía. La energía puede almacenarse de distintas formas, pero en términos prácticos, únicamente los embalses y las baterías recargables constituyen opciones interesantes.

Los desarrollos técnicos en las dos últimas décadas han abierto el camino para las más pequeñas centrales hidroeléctricas. Los nuevos desarrollos en materiales, la electrónica en estado sólido, los aparatos electrodomésticos y el alumbrado han aumentado el uso eficiente de la electricidad y han disminuido la demanda de energía. Aún más importante, más personas se sirven ahora por capacidad instalada de kilovatio de una central hidroeléctrica.
Una comparación entre los aparatos electrodomésticos de los años 50 y sus equivalentes de hoy en día muestra que las eficiencias para el mismo servicio se han duplicado; en el campo de la electrónica, el progreso se ha multiplicado por diez y en el caso del alumbrado, los desarrollos recientes prometen un aumento equivalente a seis veces. Las baterías recargables eléctricas no han cambiado mucho en 20 años, pero ahora lo estudios se proponen crear baterías que sean más livianas, más confiables y de mayor capacidad por unidad de volumen.

Desafortunadamente, la naturaleza de la demanda doméstica de energía comporta una gran dificultad en las pequeñas centrales hidroeléctricas. Su carácter desigual y de momentos de máxima demanda requiere el uso de dispositivos de control que bien eliminen o almacenen energía. La demanda desigual también requiere que el equipo instalado tenga capacidad suficiente para manejar una demanda máxima. En estas circunstancias, la mayor parte de la inversión en una central pequeña permanecerá sin ser aprovechada e improductiva.

Ha habido varios intentos de maximizar la utilidad de una central hidroeléctrica pequeña. Ello se puede logar con el mejoramiento de su factor de carga mediante la distribución de la carga en un número mayor de población. En los sitios donde los recursos son muy escasos, un poco de electricidad para todos podría ser mejor que un suministro abundante para unos pocos. La falta de conocimiento a menudo da como resultado un mal uso de la electricidad colocándola fuera del alcance de las comunidades pobres. 
Un buen ejemplo es la creencia de que se deben imponer mediante ley los estándares para los equipos eléctricos y la distribución de energía. Quizás estándares más laxos generarían ideas que podrán reducir los costos totales de la electricidad. No tiene sentido adoptar e imponer estándares que no corresponden a las condiciones locales; sería como intentar vivir con los estándares de Estados Unidos en Colombia donde el ingreso anual de muchas familias se encuentra alrededor de los USD1000. Es necesario que exista un estándar apropiado para cada situación.

El usuario final de la electricidad puede ser educado para utilizar la energía de forma eficiente si el precio de la energía es correcto. Al tiempo que educar al usuario final sería de ayuda, el diseño podría incorporar métodos para promover el uso eficiente. Tomemos, por ejemplo, el sistema tradicional de servicios públicos en los pueblos, con una red de alta tensión para la distribución, transformadores, y cableado eléctrico en el hogar según el estándar que se haya adoptado. Al sumarle un medidor y bombillas incandescentes ineficientes, el resultado es un sistema apropiado para algunas personas que tienen que comprar todo para su hogar.

Por otro lado, el mismo servicio público podría entregar, posiblemente cada tres días o en un periodo similar, una batería recargable que se enganche en el cableado de cada hogar para suministrar energía de CC eficiente para alumbrado y aparatos. Cada alternativa posee ventajas y desventajas, pero una será la más apropiada. Los desarrollos tecnológicos del futuro probablemente presentarán otras alternativas válidas. Por el momento, sin embargo, es claro que en la planeación del desarrollo de pequeñas centrales hidroeléctricas, las soluciones no convencionales deben ser evaluadas en cada fase.

\section{Diversas alternativas para proyectos de microhidroeléctricas}

Algunas opciones para proyectos de microhidroeléctricas dependen de la cantidad de energía que se vaya a explotar. El cuadro 1 muestra tan solo algunas de las posibles combinaciones para proyectos de hidroeléctricas pequeñas. Estos ejemplos excluyen intencionalmente

\begin{tabular}{|c|c|c|c|c|c|c|c|c|c|c|}
\hline \multicolumn{11}{|c|}{ CUADRO 1. Ejemplos de proyectos de microhidroeléctricas para cargas domésticas } \\
\hline Opción & $\begin{array}{l}\text { Capacidad del } \\
\text { recurso hidrico } \\
\text { fiable (kW) }\end{array}$ & $\begin{array}{c}\text { Potencia } \\
\text { del eje } \\
(\mathrm{kW})\end{array}$ & $\begin{array}{c}\text { Eficiencia } \\
\text { típica de } \\
\text { la turbina }\end{array}$ & $\begin{array}{c}\text { Eficiencia } \\
\text { típica del } \\
\text { generador }\end{array}$ & $\begin{array}{c}\text { Regulación o } \\
\text { control }\end{array}$ & $\begin{array}{c}\text { Tipo de corriente o transmisión de } \\
\text { energia }\end{array}$ & $\begin{array}{c}\text { Números } \\
\text { de } \\
\text { hogares } \\
\text { servidos }\end{array}$ & $\begin{array}{c}\text { Energía } \\
\text { productiva } \\
\text { por día } \\
\text { (kWh/día) } \\
\end{array}$ & $\begin{array}{c}\text { Capacidad } \\
\text { máxima } \\
(\mathrm{kW})\end{array}$ & $\begin{array}{c}\text { Eficiencia } \\
\text { y factor de } \\
\text { carga } \\
\text { Total } \\
\end{array}$ \\
\hline 1 & $\begin{array}{l}0.5 \\
\text { Agua fluyente }\end{array}$ & .35 & .7 & .7 & $\begin{array}{l}\text { Regulador } \\
\text { de tensión } \\
\text { electrónico }\end{array}$ & $\begin{array}{l}\text { bat } \frac{50 \mathrm{~m}}{12 \mathrm{VCC}, 20 \% \text { pérdidas }} \\
\text { bat }- \text { Conv. } \frac{500 \mathrm{~m}}{120 \mathrm{VCA}, 30 \% \text { pérdidas }}\end{array}$ & $1-2$ & 5.0 & $\begin{array}{l}2.0 \\
1.0\end{array}$ & $\begin{array}{l}0.4 \\
\\
0.35 \\
\text { alto }\end{array}$ \\
\hline 2 & $\begin{array}{l}5 \\
\text { Agua fluyente }\end{array}$ & 3.5 & .7 & .75 & $\begin{array}{l}120 \text { VCA } 12 \\
\text { VCC } \\
\text { Control de } \\
\text { tensión } \\
\end{array}$ & $\begin{array}{l}\text { Las baterías son transportadas y } \\
\text { distribuidas, } 20 \% \text { pérdida }\end{array}$ & $10-20$ & 50 & 20 & $\begin{array}{l}0.4 \\
\text { alto }\end{array}$ \\
\hline 3 & $\begin{array}{l}5 \\
\text { Embalse de } \\
\text { almacenamiento }\end{array}$ & 25 & .7 & .8 & $\begin{array}{l}\text { Control de } \\
\text { flujo } \\
\text { mecánico } \\
\text { (Woodward) }\end{array}$ & $\frac{500 \mathrm{~m}}{\text { Baja tensión CA }}$ & $12-24$ & $\begin{array}{l}60 \text { o más } \\
\text { si el flujo } \\
\text { lo } \\
\text { permite }\end{array}$ & 20 & $\begin{array}{l}0.5 \\
\text { bajo }\end{array}$ \\
\hline 4 & $\begin{array}{l}5 \\
\text { Agua fluyente }\end{array}$ & 3.5 & .7 & .8 & Sin control & $\frac{500 \mathrm{~m}}{\text { Baja tensión CA }}$ & 2 & 60 & 2.5 & $\begin{array}{l}0.5 \\
\text { Bajo } \\
\end{array}$ \\
\hline 5 & $\begin{array}{l}2-20 \\
\text { Agua fluyente }\end{array}$ & $\begin{array}{l}1.5 \mathrm{a} \\
15\end{array}$ & .7 & .8 & $\begin{array}{l}\text { Lastre y } \\
\text { control de } \\
\text { carga } \\
\text { electrónicos } \\
\end{array}$ & $\frac{500 \mathrm{~m}}{\text { Baja tensión CA }}$ & $1-15$ & $25-250$ & 10 & $\begin{array}{l}0.55 \\
\text { bajo }\end{array}$ \\
\hline 6 & $\begin{array}{l}2-20 \\
\text { Agua fluyente }\end{array}$ & $\begin{array}{l}1.5 \mathrm{a} \\
15\end{array}$ & .7 & .8 & $\begin{array}{l}\text { Lastre y } \\
\text { control de } \\
\text { carga } \\
\text { electrónicos }\end{array}$ & $\begin{array}{l}\text { Cargador de batería y baterías a } \\
\text { distribuir } \\
\qquad \frac{500 \mathrm{~m}}{\text { Baja tensión CA }}\end{array}$ & $4-40$ & $20-200$ & $2-100$ & $\begin{array}{l}0.4 \\
\text { alto }\end{array}$ \\
\hline 7 & $\begin{array}{l}20-500 \\
\text { Agua fluyente }\end{array}$ & & .8 & .9 & $\begin{array}{l}\text { Lastre y } \\
\text { control de } \\
\text { carga } \\
\text { electrónicos }\end{array}$ & 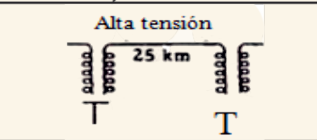 & $\begin{array}{l}30- \\
5000\end{array}$ & $300-7500$ & $12-350$ & $\begin{array}{l}0.64 \\
\text { bajo }\end{array}$ \\
\hline
\end{tabular}


usos productivos finales, porque tales actividades a menudo resultana partir deesfuerzos deemprendimiento y comunitarios en sociedades libres. En sociedades con organizaciones comunales, es indispensable la debida consideración del aspecto productivo de la central durante la fase de diseño. Inicialmente, se le debe dar prioridad a la carga doméstica en dichos casos, porque la carga industrial generalmente se promoverá por sí sola una vez el know-how tecnológico esté disponible.

Con la opción 1, el costo puede minimizarse solo si los usuarios finales interesados explotan la potencia instalada. El principal problema de esta opción es encontrar una unidad generadora hídrica económica, confiable y flexible que cualquiera pueda instalar; solo hasta hace poco se han realizado algunos esfuerzos para comercializar dichos dispositivos. El enfoque inicial es utilizar alternadores automotrices estándar y turbinas fabricadas localmente. Los reguladores electrónicos confiables se han utilizado para regular la tensión con el objetivo de cargar las baterías sin dañarlas.

En pocas palabras, el enfoque de la Opción 1 requiere la disponibilidad de una unidad generadora hidroeléctrica que se compre como se compraría una pieza de equipo agrícola y que se utilice para suministrar energía para alumbrado, comunicaciones y algunos pocos aparatos del hogar. Los inversores electrónicos a un precio razonable permitirían que la energía se transmita al usuario final con una tensión más alta; no obstante, las pérdidas y la capacidad reducida limitan de alguna manera la idoneidad de estas unidades hidroeléctricas muy pequeñas.

Las opciones 2, 3 y 4 promueven el mismo recurso pero difieren ampliamente en el número de personas a quienes se satisface y los costos implicados. La opción 2 se enfoca en la demanda de energía y es económica pero depende de alguna forma de un transporte práctico para las baterías y el control de su carga. Los factores de carga de las centrales correspondientes a las Opciones 3 y 4 muestran que serán más caras por usuario potencial. La Opción 3 busca adaptar la tecnología de las grandes hidroeléctricas a los desarrollos de las pequeñas. Con esta opción, el factor de carga real de la central, algunas veces, puede ser alto, pero las inversiones extra permanecerán sin ser aprovechadas parte del tiempo porque el diseño se enfoca en satisfacer la demanda máxima de energía. Cuando los fondos son escasos, la Opción 4 puede ser una buena solución solo si se encuentra algún uso productivo para la energía de forma que aumente el factor de carga.

Las Opciones 5 y 6 muestran las ventajas de intentar enfoques no convencionales para suministrar electricidad a pequeñas aldeas. El mayor beneficio de la Opción 6 es el número mayor de personas que pueden servirse de una determinada fuente fiable. Así como la Opción 2, la Opción 6 implica el uso de baterías que pueden presentar dificultades de manejo.

Ninguna de estas opciones puede permitirse estudios de ingeniería costosos. Estas opciones requieren el uso de la autoayuda comunitaria, el know-how local y las tecnologías locales.

La Opción 7 es una central microhidroeléctrica tradicional para condiciones de recursos hidroeléctricos abundantes. La única diferencia entre esta central y una central equivalente del nuevo siglo es el tipo de controles. El regulador de carga electrónico reciente desarrollado específicamente para microhidroeléctricas recibe la corriente del generador y cambia la corriente no utilizada por el consumidor a una carga de lastre para garantizar que una carga constante sea puesta en la turbina. Los costos son menores que los de los controles electromecánicos convencionales, y la confiabilidad es mucho mayor. De hecho, el regulador de carga electrónico mejora la confiabilidad de la central drásticamente y obtiene asimismo algo de ahorro en los costos. Las únicas partes móviles son la turbina y el generador; las válvulas o compuertas de control no se requieren porque el flujo es constante; y la protección contra sobretensiones no es necesaria.

Los dispositivos de seguridad para exceso de velocidad se pueden instalar en caso de que la carga en el turbogenerador se pierda accidentalmente; por lo tanto, las turbinas pueden contar con geometría fija que incorpore la flexibilidad para hacer frente a un rango de presiones y flujos. Los generadores también pueden simplificarse un poco para hacer las funciones de regulación automática dentro de la máquina innecesarias. 